\title{
INTERSECTIONS OF $\Gamma$-ISOTYPE SUBGROUPS IN ABELIAN GROUPS
}

\author{
JINDǨICH BEČVÁR
}

\begin{abstract}
A subgroup $H$ of an abelian group $G$ is an intersection of isotype subgroups of $G$ if and only if, for each prime $p$, if $x+H$ is a coset of order $p$ then there is another coset of order $p$ containing an element $y$ of order $p$ such that $h_{p}^{*}(x) \leqslant h_{p}^{*}(y)$. A subgroup $H$ of $G$ is isotype in $G$ if and only if, for each prime $p$, every coset $x+H$ of order $p$ contains an element $y$ of order $p$ such that $h_{p}^{*}(x) \leqslant$ $h_{p}^{*}(y)$.
\end{abstract}

1. Introduction. All groups in this paper are abelian, we shall follow the notation and terminology of [3]. Let $G$ be a group. A subgroup $H$ of $G$ is said to be $p$-isotype in $G$ if $p^{\beta} H=H \cap p^{\beta} G$ for each ordinal $\beta ; H$ is called isotype in $G$ if $H$ is $p$-isotype in $G$ for each prime $p$. Let $\mathbf{P}$ be the set of all primes and $\Gamma=\left(\alpha_{p}\right)_{p \in \mathbf{P}}$ a sequence, where each $\alpha_{p}$ is either an ordinal or the symbol $\infty$ which is considered to be larger than any ordinal. A subgroup $H$ is said to be $\Gamma$-isotype in $G$ if $p^{\beta} H=H \cap p^{\beta} G$ for every prime $p$ and for every ordinal $\beta \leqslant \alpha_{p}$. If $\alpha_{p}=0,1, \omega, \infty$ for every prime $p$ then $\Gamma$-isotype subgroups are precisely subgroups, neat subgroups, pure subgroups, isotype subgroups respectively. Further, $G_{t}$ and $G_{p}$ are the torsion part of $G$ and the $p$-component of $G_{t}$. It is natural to use the symbol $p^{\alpha} G[p]$.

K. M. Rangaswamy [7] obtained a characterization of intersections of neat subgroups. L. Fuchs [4, 5] asked for a characterization of those subgroups that are an intersection of pure subgroups. C. Megibben [6] answered this question for p-groups and D. Boyer and K. M. Rangaswamy [1] for arbitrary abelian groups.

The purpose of this paper is to describe those subgroups that are an intersection of $\Gamma$-isotype subgroups (see Theorem 1). As corollaries yield a characterization of intersections of isotype subgroups (see Corollary 2) and the recalled results of Boyer and Rangaswamy [1] and Rangaswamy [7] (see Corollaries 3 and 4). Finally, $\Gamma$-isotype and isotype subgroups are characterized (see Theorem 2 and Corollary 5).

In the papers [6] and [1], the given subgroup is realized as an intersection of neat subgroups which are then shown to be pure. We do not follow this idea in the present paper. To prove that the given subgroup $H$ is an intersection of $\Gamma$-isotype subgroups, we construct for each element $x \in G \backslash H$ a $\Gamma$-isotype subgroup containing $H$ but not including $x$.

The author is indebted to Professor Rangaswamy for sending him a preprint of the paper [1]. It was the reading of this paper which inspired the presented work.

Received by the editors July 27, 1981 and, in revised form, April 7, 1982.

1980 Mathematics Subject Classification. Primary 20K99.

Key words and phrases. Abelian groups, isotype and $\Gamma$-isotype subgroups.

(C)1982 American Mathematical Society 0002-9939/82/0000-0367/\$02.50 
The author wishes to express his gratitude to the referee for his advice how to make the main proof more readable.

\section{Construction of $\Gamma$-isotype and isotype subgroups.}

LEMMA 1. Let $G$ be a group, $x \in G$ and $p$ a prime. If $K$ is a subgroup of $G$ maximal with respect to the conditions $p x \in K$ and $x \notin K$ then $K$ is q-isotype in $G$ for each prime $q \neq p$. Moreover, if $x \in p^{\beta} G$ and $p x \in p^{\beta+1} K$ for some ordinal $\beta$, then $p^{\alpha} K=K \cap p^{\alpha} G$ for each ordinal $\alpha \leqslant \beta+1$.

Proof. Suppose $g \in G \backslash K$ and $q g \in K$. Since $x \in\langle g, K\rangle, x=n g+k$, where $k \in K$ and $n$ is an integer; obviously $(n, q)=1$. Hence png $\in K$ and $q \mid p n$. This contradiction implies that $(G / K)_{q}=0$ and hence $K$ is $q$-isotype in $G$ (see [3, Lemma 103.1]).

Moreover suppose that $x \in p^{\beta} G$ and $p x \in p^{\beta+1} K$ for some ordinal $\beta$. We prove that $p^{\alpha} K=K \cap p^{\alpha} G$ for each ordinal $\alpha \leqslant \beta+1$. It is sufficient to show that if this equality holds for $\alpha \leqslant \beta$ then it holds for $\alpha+1$. Let $k \in K \cap p^{\alpha+1} G$, i.e. $k=p g$, where $g \in p^{\alpha} G$; obviously $k \in K \cap p^{\alpha} G=p^{\alpha} K$. If $g \in K$ then $g \in K \cap p^{\alpha} G=p^{\alpha} K$ and $k=p g \in p^{\alpha+1} K$. If $g \notin K$ then $x \in\langle g, K\rangle$, i.e. $x=n g+\bar{k}$, where $\bar{k} \in K$ and $\boldsymbol{n}$ is an integer; obviously $(p, n)=1$. Since $g \in p^{\alpha} G$ and $x \in p^{\beta} G \subset p^{\alpha} G$, we have $\bar{k} \in K \cap p^{\alpha} G=p^{\alpha} K$. Now, $n k=p n g=p x-p \bar{k} \in p^{\alpha+1} K$. Since $(p, n)=1, k \in$ $p^{\alpha+1} K$.

LEMMA 2. Let $H$ be a subgroup of a group $G, p$ a prime, $\beta$ an ordinal or the symbol $\infty$ and $x \in G$. Let $A$ be a subset of $G$ such that for every ordinal $\gamma<\beta$ there is an element $a \in A$ with $a \in\left\langle H, p^{\gamma} G[p], p x\right\rangle$. If $K$ is a subgroup of $G$ maximal with respect to the properties $x \notin K$ and $K \supset\langle H, p x,\{x-a ; a \in A\}\rangle$ then $K$ is q-isotype in $G$ for each prime $q \neq p$ and $p^{\gamma} K=K \cap p^{\gamma} G$ for each ordinal $\gamma \leqslant \beta$. Moreover, if $K \supset p^{\beta} G$ then $K$ is isotype in $G$.

Proof. With respect to Lemma 1 , it is sufficient to show that if $p^{\gamma} K=K \cap p^{\gamma} G$ for $\gamma<\beta$ then $p^{\gamma+1} K=K \cap p^{\gamma+1} G$. Let $k \in K \cap p^{\gamma+1} G$, i.e. $k=p g$, where $g \in p^{\gamma} G$; obviously $k \in K \cap p^{\gamma} G=p^{\gamma} K$. If $g \in K$ then $k=p g \in p^{\gamma+1} K$. If $g \notin K$ then $x \in\langle g, K\rangle$, i.e. $x=n g+\bar{k}$, where $\bar{k} \in K$ and $n$ is an integer. Obviously $(p, n)=1$ and $x-\bar{k} \in p^{\gamma} G$. Now, $n k=p n g=p(x-\bar{k})$. By assumption, there is an element $a \in A$ such that $a=h+\bar{g}+m p x$, where $h \in H, \bar{g} \in p^{\gamma} G[p]$ and $m$ is an integer. Consequently, $\overline{\boldsymbol{g}}=a-h-m p x, x-\bar{g}-\bar{k}=(x-a)+h+m p x-\bar{k} \in K \cap p^{\gamma} G$ $=p^{\gamma} K$ and hence $n k=p(x-\bar{g}-\bar{k}) \in p^{\gamma+1} K$. Since $(p, n)=1, k \in p^{\gamma+1} K$. Moreover, if $K \supset p^{\beta} G$ then $p^{\beta} G=p^{\beta} G \cap K=p^{\beta} K$ and hence $p^{\gamma} G \cap K=p^{\gamma} K$ for each ordinal $\gamma$.

COROLlaRY 1. Let $H$ be a subgroup of a group $G, p$ a prime, $\beta$ an ordinal or the symbol $\infty$ and $x \in G$. Let $x \in\left\langle H, p^{\gamma} G[p], p x\right\rangle$ for each ordinal $\gamma<\beta$. If $K$ is $a$ subgroup of $G$ maximal with respect to the properties $x \notin K$ and $K \supset\langle H, p x\rangle$ then $K$ is $q$-isotype in $G$ for each prime $q \neq p$ and $p^{\gamma} K=K \cap p^{\gamma} G$ for each ordinal $\gamma \leqslant \beta$. Moreover, if $K \supset p^{\beta} G$ then $K$ is isotype in $G$.

Proof. Put $A=\{x\}$ and the assertion follows from Lemma 2. 
LEMMA 3. Let $H$ be a subgroup of a group $G$ and $\beta$ an ordinal or the symbol $\infty$ such that

(i) $\forall \gamma<\beta \exists a \in p^{\gamma} G[p] \backslash H$.

(ii) $\forall a \in G[p] \backslash H \exists \gamma<\beta a \notin H+p^{\gamma} G[p]$.

Then for each ordinal $\mu<\beta$ there is a subset $A$ of $p^{\mu} G[p]$ such that

(a) $\forall \gamma<\beta \exists a \in A a \in p^{\gamma} G[p]$,

(b) for each finite subset $A^{\prime}$ of $A$ there are an element $a \in A^{\prime}$ and an ordinal $\mu<\nu<\beta$ with $A^{\prime} \backslash\{a\} \subset p^{\nu} G[p]$ and $a \notin H+p^{\nu} G[p]$.

Proof. First note that $\beta$ is a limit ordinal. For, otherwise there is an element $a \in p^{\beta-1} G[p] \backslash H$ (resp. $a \in p^{\infty} G[p] \backslash H$ ) by (i), and we have a contradiction with (ii).

Let $\varepsilon$ be the least ordinal such that $\beta$ is cofinal with $\varepsilon($ i.e. $\varepsilon=\operatorname{cof}(\beta))$. Let $\beta_{\alpha}$, $\alpha<\varepsilon$ be an ascending sequence of ordinals with the limit $\beta$. For each ordinal $\alpha<\varepsilon$ let $a_{\alpha} \in p^{\beta_{\alpha}} G[p] \backslash H$ (see (i)) and further let $\gamma_{\alpha}$ be the least ordinal such that $a_{\alpha} \notin H+p^{\gamma_{\alpha}} G[p]$ (see (ii)); obviously $\beta_{\alpha}<\gamma_{\alpha}<\beta$. We use the transfinite induction to define an ascending sequence of ordinals $\delta_{\alpha}, \alpha<\varepsilon$ with the following properties

$$
\begin{gathered}
\alpha \leqslant \delta_{\alpha}<\varepsilon, \\
\forall \alpha^{\prime}<\alpha \gamma_{\delta_{\alpha^{\prime}}}<\beta_{\delta_{\alpha^{\prime}}} .
\end{gathered}
$$

Put $\delta_{0}=0,(1,0)$ and $(2,0)$ hold.

Suppose that for an ordinal $\alpha<\varepsilon$, the ordinal $\delta_{\alpha}$ with the properties $(1, \alpha)$ and $(2, \alpha)$ is defined. Let $\delta_{\alpha+1}$ be the least ordinal $\kappa$ such that $\gamma_{\delta_{\alpha}}<\beta_{\kappa}$. Obviously $(1, \alpha+1)$ and $(2, \alpha+1)$ hold.

Suppose that $\alpha<\varepsilon$ is a limit ordinal and for each ordinal $\alpha^{\prime}<\alpha$, the ordinal $\delta_{\alpha^{\prime}}$ with the properties $\left(1, \alpha^{\prime}\right)$ and $\left(2, \alpha^{\prime}\right)$ is defined. Since $\varepsilon=\operatorname{cof}(\beta)$, there is an ordinal $\kappa<\varepsilon$ such that $\gamma_{\delta_{\alpha^{\prime}}}<\beta_{\kappa}$ for each $\alpha^{\prime}<\alpha$; let $\delta_{\alpha}$ be the least such ordinal $\kappa$. Obviously $(1, \alpha)$ and $(2, \alpha)$ hold.

Since $\alpha \leqslant \delta_{\alpha}<\varepsilon$, we have $\beta_{\alpha} \leqslant \beta_{\delta_{\alpha}}<\beta$ and $\beta$ is the limit of the sequence $\beta_{\delta_{\alpha}}$, $\alpha<\varepsilon$. Put $A=\left\{a_{\delta_{\alpha}} ; \alpha<\varepsilon\right.$ and $\left.\mu<\beta_{\delta_{\alpha}}\right\}$. Obviously, $A$ is a subset of $p^{\mu} G$ [ $p$ ]. If $A^{\prime}$ is a finite subset of $A$ then the element $a_{\delta_{\alpha}} \in A^{\prime}$ with the least index $\alpha$ and the ordinal $\nu=\gamma_{\delta_{\alpha}}$ have the desired properties.

\section{The main theorem and corollaries.}

THEOREM 1. Let $H$ be a subgroup of a group $G$ and $\Gamma=\left(\alpha_{p}\right)_{p \in P}$. The following are equivalent:

(i) $H$ is an intersection of $\Gamma$-isotype subgroups.

(ii) For all primes $p$ and all ordinals $\alpha<\alpha_{p}$, if there is an element $x \in p^{\alpha} G \backslash H$ with $p x \in H$ then there is an element $y \in p^{\alpha} G[p] \backslash H$.

(iii) For each prime $p$, if $\beta$ is the least ordinal such that $p^{\beta} G[p] \subset H$ and $\beta<\alpha_{p}$ then for each element $x \in G \backslash H$ with $p x \in H, x \notin H+p^{\beta} G$.

Proof. (i) $\rightarrow$ (ii). Suppose that for some prime $p$ and some ordinal $\alpha<\alpha_{p}$, $x \in p^{\alpha} G \backslash H$ and $p x \in H$. Let $K$ be a $\Gamma$-isotype subgroup of $G$ such that $H \subset K$ and 
$x \notin K$. Since $p x \in K \cap p^{\alpha+1} G=p^{\alpha+1} K$, there is an element $k \in p^{\alpha} K$ such that $p x=p k$. Now, $x-k \in p^{\alpha} G[p] \backslash H$.

(ii) $\rightarrow$ (iii). Let $\beta$ be the least ordinal such that $p^{\beta} G[p] \subset H$ and $\beta<\alpha_{p}$, let $x \in G \backslash H$ and $p x \in H$. If $x=h+z$, where $h \in H$ and $z \in p^{\beta} G$, then $z \in p^{\beta} G \backslash H$, $p z \in H$ and (ii) yields a contradiction. Hence $x \notin H+p^{\beta} G$.

(iii) $\rightarrow$ (i). Let $x \in G \backslash H$ and $p x \in H$ for some prime $p$. We wish to construct a $\Gamma$-isotype subgroup $K$ of $G$ such that $x \notin K$ and $H \subset K$. We distinguish two cases:

Case I. $p^{\gamma} G[p] \subset H$ for some $\gamma<\alpha_{p}$. In this case denote by $\beta$ the least ordinal such that $p^{\beta} G[p] \subset H$. Hence $\beta<\alpha_{p}$.

Case II: $p^{\gamma} G[p] \not \subset H$ for each ordinal $\gamma<\alpha_{p}$. In this case put $\beta=\alpha_{p}$.

In the both cases we have:

$$
\forall \gamma<\beta \quad \exists a \in p^{\gamma} G[p] \backslash H .
$$

Suppose that $x \in H+p^{\gamma} G[p]$ for each ordinal $\gamma<\beta$. In case I we have $x \notin H+$ $p^{\beta} G$ by (iii). Let $K$ be a subgroup of $G$ maximal with respect to $x \notin K$ and further that $H+p^{\beta} G \subset K$, in case I and $H \subset K$, in case II. By Corollary $1, K$ is isotype in $G$ in case $I$ and $\Gamma$-isotype in $G$ in case II.

Now suppose that $x \notin H+p^{\mu} G[p]$ for some ordinal $\mu<\beta$. We consider two cases to define a certain subset $A$ of $G[p]$.

Case 1. $\exists a \in G[p] \backslash H \forall \gamma<\beta, a \in H+p^{\gamma} G[p]$. Put $A=\{a\}$.

Case 2. $\forall a \in G[p] \backslash H \exists \gamma<\beta, a \notin H+p^{\gamma} G[p]$. Let $A$ be the subset of $p^{\mu} G[p]$ from Lemma 3.

In the both cases 1 and 2 we have:

$$
\forall \gamma<\beta \quad \exists a \in A a \in H+p^{\gamma} G[p] .
$$

Define

$$
L= \begin{cases}H+p^{\beta} G+\langle\{x-a ; a \in A\}\rangle, & \text { in case I } \\ H+\langle\{x-a ; a \in A\}\rangle, & \text { in case II. }\end{cases}
$$

If $x \notin L$ then a subgroup $K$ of $G$ maximal with respect to the properties $L \subset K$, $x \notin K$ will be by Lemma 2, isotype in case I and $\Gamma$-isotype in case II.

Suppose $x \in L$. We claim case 2 does not arise at all. Suppose on the contrary, case 2 arises. In this case we can write

$$
x= \begin{cases}h+g+\sum_{i=1}^{r} n_{i}\left(x-a_{i}\right), & \text { in case I } \\ h+\sum_{i=1}^{r} n_{i}\left(x-a_{i}\right), & \text { in case II, }\end{cases}
$$

where $h \in H, g \in p^{\beta} G, a_{i} \in A, n_{i}$ are integers and $\left(n_{i}, p\right)=1$. By Lemma 3, we may assume that there is an ordinal $\nu$ with $\mu<\nu<\beta, a_{1} \notin H+p^{\nu} G[p]$ and $a_{2}, \ldots, a_{r} \in p^{\nu} G[p]$. Now, $p x \in H$ implies, in case I, that $p g \in H$ and our hypothesis (iii) implies that $g \in H$. Thus in both cases I and II we conclude that

$$
\left(1-\sum_{i=1}^{r} n_{i}\right) \cdot x \in H+p^{\mu} G[p] .
$$


Since $x \notin H+p^{\mu} G[p]$, then $p \mid\left(1-\sum n_{i}\right)$ and this gives that $\Sigma n_{i} a_{i} \in H$. Then $n_{1} a_{1} \in H+p^{\nu} G[p]$, a contradiction. Thus when $x \in L$, case 1 prevails so that $A=\{a\}$ with the element $a$ having the state properties. In case I, $a \notin H+p^{\beta} G$ by our hypothesis (iii). Now choose $K$ maximal with respect to $a \notin K$ and further that $K \supset H+p^{\beta} G$, in case I and $K \supset H$, in case II. Then, by Corollary $1, K$ is isotype in $G$ in case $I$ and $\Gamma$-isotype in $G$ in case II. Since

$$
x= \begin{cases}h+g+n(x-a), & \text { in case I } \\ h+n(x-a), & \text { in case II, }\end{cases}
$$

where $h \in H, g \in p^{\beta} G, n$ is an integer and $(n, p)=1$, it is easy to see that $x \notin K$. This proves (iii) $\rightarrow$ (i).

COROLlARY 2. Let $H$ be a subgroup of a group $G$. The following are equivalent:

(i) $H$ is an intersection of isotype subgroups.

(ii) For all primes $p$ and all ordinals $\alpha$, if there is an element $x \in p^{\alpha} G \backslash H$ with $p x \in H$ then there is an element $y \in p^{\alpha} G[p] \backslash H$.

(iii) For each prime $p$ and each coset $x+H$ of order $p$, there is another coset $y+H$ of order $p$ such that $y$ is of order $p$ and $h_{p}^{*}(x) \leqslant h_{p}^{*}(y)$.

(iv) For each prime $p$, each natural number $n$ and each coset $x+H$ of order $p^{n}$, there is another coset $y+H$ of order $p^{n}$ such that $y$ is of order $p^{n}$ and $h_{p}^{*}(x) \leqslant h_{p}^{*}(y)$.

Proof. By Theorem 1, (i) and (ii) are equivalent. The equivalence of assertions (ii), (iii) and the implication (iv) $\rightarrow$ (iii) are obvious. The proof of the implication (iii) $\rightarrow$ (iv) follows by induction on $n$.

COROLlARY 3 (BOYER AND RANGASWAMY [1]). Let $H$ be a subgroup of a group $G$. The following are equivalent:

(i) $H$ is an intersection of pure subgroups.

(ii) For all primes $p$ and all natural numbers $n$, if there is an element $x \in p^{n} G \backslash H$ with $p x \in H$ then there is an element $y \in p^{n} G[p] \backslash H$.

(iii) For all primes $p$ and all natural numbers $n$, if there is an element $g \in G$ such that $p^{n} g \in H$ and $p^{n-1} g \notin H$ then there is an element $z \in G$ such that $p^{n} z=0$ and $p^{n-1} z \notin H$.

CoROllaRY 4 (RANGASWAMY [7]). Let $H$ be a subgroup of a group $G$. The following are equivalent:

(i) $H$ is an intersection of neat subgroups.

(ii) For all primes $p$, if there is an element $x \in G \backslash H$ with $p x \in H$ then there is an element $y \in G[p] \backslash H$.

4. $\Gamma$-isotype and isotype subgroups. In conclusion, it is interesting to give a following characterization of $\Gamma$-isotype subgroups.

THEOREM 2. Let $H$ be a subgroup of a group $G, \Gamma=\left(\alpha_{p}\right)_{p \in \mathbf{P}}$. The following are equivalent:

(i) $H$ is $\Gamma$-isotype in $G$.

(ii) For each prime $p$ and each ordinal $\alpha<\alpha_{p}$, if there is an element $x \in p^{\alpha} G \backslash H$ such that $p x \in H$ then there is an element $y \in p^{\alpha} G[p] \backslash H$ such that $x-y \in H$. 
Proof. (i) $\rightarrow$ (ii). Suppose that for some prime $p$ and some ordinal $\alpha<\alpha_{p}$, $x \in p^{\alpha} G \backslash H$ and $p x \in H$. Hence $p x \in H \cap p^{\alpha+1} G=p^{\alpha+1} H$ and there is an element $h \in p^{\alpha} H$ such that $p x=p h$. Now, $y=x-h \in p^{\alpha} G[p] \backslash H$ and $x-y \in H$.

(ii) $\rightarrow$ (i). It is sufficient to show that for each prime $p$ and each ordinal $\alpha<\alpha_{p}$, the equality $p^{\alpha} G \cap H=p^{\alpha} H$ implies the equality $p^{\alpha+1} G \cap H=p^{\alpha+1} H$. Let $h \in$ $p^{\alpha+1} G \cap H$, i.e. $h=p g$, where $g \in p^{\alpha} G$. If $g \in H$ then $h \in p^{\alpha+1} H$. If $g \notin H$ then by (ii), there is an element $y \in p^{\alpha} G[p] \backslash H$ such that $g-y \in H$. Now, $g-y \in p^{\alpha} G \cap$ $H=p^{\alpha} H$ and hence $p(g-y)=h \in p^{\alpha+1} H$.

COROLLARY 5. Let $H$ be a subgroup of a group $G$. The following are equivalent:

(i) $H$ is isotype in $G$.

(ii) For each prime $p$, every coset $x+H$ of order $p$ contains an element $y$ of order $p$ such that $h_{p}^{*}(x) \leqslant h_{p}^{*}(y)$.

(iii) For each prime $p$ and each natural number $n$, every coset $x+H$ of order $p^{n}$ contains an element $y$ of order $p^{n}$ such that $h_{p}^{*}(x) \leqslant h_{p}^{*}(y)$.

Proof. By Theorem 2, (i) and (ii) are equivalent. Obviously (iii) implies (ii). We use the induction to prove the implication (ii) $\rightarrow$ (iii). Suppose that (iii) holds for $n \geqslant 1$. Let $x+H$ be a coset of order $p^{n+1}$ and $h_{p}^{*}(x)=\alpha$. Since $p x+H$ is a coset of order $p^{n}$, there is an element $y_{1}$ of order $p^{n}$ such that $y_{1}-p x \in H$ and $\alpha+1 \leqslant$ $h_{p}^{*}(p x) \leqslant h_{p}^{*}\left(y_{1}\right)$. Write $y_{1}=p y_{2}$, where $y_{2} \in p^{\alpha} G$. If $y_{2}-x \in H$ the we are through, since $y_{2}$ is of order $p^{n+1}$ and $h_{p}^{*}(x) \leqslant h_{p}^{*}\left(y_{2}\right)$. If $y_{2}-x \notin H$ then by (ii), the coset $y_{2}-x+H$ of order $p$ contains an element $y_{3}$ of order $p$ such that $\alpha \leqslant h_{p}^{*}\left(y_{2}-x\right) \leqslant$ $h_{p}^{*}\left(y_{3}\right)$. Finally, $y_{2}-y_{3}$ is of order $p^{n+1}, y_{2}-y_{3}-x \in H$ and

$$
\alpha=h_{p}^{*}(x) \leqslant h_{p}^{*}\left(y_{2}-y_{3}\right) \text {. }
$$

\section{REFERENCES}

1. D. Boyer and K. M. Rangaswamy, Intersections of pure subgroups in abelian groups, Proc. Amer. Math. Soc. 81 (1981), 178-180.

2. B. Charles, Une caractérisation des intersections de sous-groups divisibles, C. R. Acad. Sci. Paris Sér. A-B 250 (1960), 256-257.

3. L. Fuchs, Infinite abelian groups. I, II, Academic Press, New York, 1970, 1973.

4. Review of [2], Math. Rev. 22 (1961), 10-11.

5. Co., Glenview, Ill., 1963, pp. 9-40.

6. C. Megibben, On subgroups of primary abelian groups, Publ. Math. Debrecen 12 (1965), 293-294.

7. K. M. Rangaswamy, Characterization of intersections of neat subgroups of abelian groups, J. Indian Math. Soc. 29 (1965), 31-36.

Department of Mathematics, Charles University, Prague, Praha 8, Sokolovská 83, Czechoslovakia 